\title{
Epigenetic silencing of tumor suppressor long non-coding RNA BM742401 in chronic lymphocytic leukemia
}

\author{
Lu Qian Wang ${ }^{1}$, Kwan Yeung Wong ${ }^{1}$, Zhen Hai Li ${ }^{1}$, Chor Sang Chim ${ }^{1}$ \\ ${ }^{1}$ Department of Medicine, Queen Mary Hospital, The University of Hong Kong, Hong Kong \\ Correspondence to: Chor Sang Chim, email: jcschim@hku.hk \\ Keywords: BM742401, InCRNA, tumor suppressor, DNA methylation, chronic lymphocytic leukemia
}

Received: January 20, $2016 \quad$ Accepted: September 20, $2016 \quad$ Published: September 26, 2016

\section{ABSTRACT}

BM742401 is a tumor suppressor IncRNA downregulated in gastric cancer. As the promoter region and the entire transcript are embedded in a CpG island, we postulated that BM742401 is a tumor suppressor IncRNA inactivated by DNA methylation in chronic lymphocytic leukemia (CLL). The promoter of BM742401 was unmethylated in normal controls including three each of normal bone marrow, peripheral blood buffy coats, and CD19-sorted peripheral B-cells, but methylated in four $(57.1 \%)$ CLL cell lines. Methylation of BM742401 correlated inversely with expression. In the completely methylated WAC3CD5+ CLL cells, 5-Aza-2'-deoxycytidine treatment led to promoter demethylation and re-expression of BM742401 transcript. Functionally, stable overexpression of BM742401 resulted in inhibition of cellular proliferation and enhanced apoptosis through caspase-9-dependent intrinsic but not caspase8-dependent extrinsic apoptosis pathway, suggesting a tumor suppressor role of BM742401 in CLL. In primary CLL samples, methylation of BM742401 was detected in 43/98 (43.9\%) of patients. Moreover, among CLL patients with standard-risk cytogenetic aberrations, methylation of BM742401 correlated with advanced Rai stage ( $\geq$ stage 2$)(P=0.002)$. Furthermore, $B M 742401$ methylation was associated with miR-129-2 methylation $(P=0.05)$. BM742401 is a tumor suppressor IncRNA frequently methylated in CLL. The mechanism of BM742401 as a tumor suppressor warrants further studies.

\section{INTRODUCTION}

DNA methylation refers to the addition of a methyl group $\left(-\mathrm{CH}_{3}\right)$ to the carbon 5 position of the cytosine ring in a $\mathrm{CpG}$ dinucleotide, leading to the formation of 5-methylcytosine [1]. Global DNA hypomethylation but aberrant DNA hypermethylation of tumor suppressor genes (TSGs) is a hallmark of many human cancers [2]. To date, in hematological cancers, methylation-mediated silencing of multiple TSGs have been found involved in the dysregulation of multiple cellular pathways including $C D K N 2 A$ and $C D K N 2 B$ in the regulation of cell-cycle, $D A P K 1$ and APAF1 in the regulation of apoptosis, and soluble $W N T$ inhibitors involved in inhibition of WNT signaling [3-6]. Of note, methylation-mediated silencing of both protein-coding TSGs, including DAPK1, ID4 and $S F R P 1$, and tumor suppressor miRNAs, including $m i R-9-3$, $m i R-34 b / c, m i R-129-2, m i R-203$ and $m i R-3151$, have been implicated in CLL leukemogenesis or prognosis [7-13].
Long non-coding RNA (lncRNA) is defined as a novel class of RNAs longer than 200 nucleotides with little or no protein-coding capacity. It can be further be categorized into five subgroups, including intergenic, intragenic, natural antisense transcripts, pseudogenes and unclassified transcripts, based on the location relative to annotated protein-coding genes (PCGs) $[14,15]$. Moreover, lncRNAs are known to activate or inhibit gene expression involved in multiple biological processes through epigenetic, transcriptional or post-transcriptional mechanisms $[16,17]$.

In addition to PCGs and miRNAs, lncRNAs are found dysregulated in many human cancers, indicating their potential oncogenic or tumor suppressor role in carcinogenesis [15]. For example, HOX transcript antisense intergenic RNA (HOTAIR) is found overexpressed in breast cancer, in particular in patients with metastasis, suggestive of an oncogenic role in cancer metastasis [18]. Conversely, maternally expressed gene 
3 (MEG3) is a tumor suppressor lncRNA downregulated in multiple human cancers, inhibiting cell proliferation through accumulation of TP53 protein by abrogating MDM2 function [19, 20]. Intriguingly, the promoterassociated $\mathrm{CpG}$ island of $M E G 3$ was found frequently hypermethylated in both solid and hematological malignancies, indicating that methylation-mediated silencing may serve as an alternative mechanism for inactivation of tumor suppressive lncRNAs [19].

In CLL, deletion of chromosome $13 \mathrm{q} 14$ is the most frequent chromosomal alteration detected in approximately $60 \%$ newly diagnosed patients, portending superior survivals [21]. In addition to $m i R-15 a / 16-1$ cluster, a lncRNA, DLEU2 was found co-deleted in the minimally deleted region (DMR) of $\operatorname{del}(13 q 14)$, hence implicating a potential tumor suppressor role of DLEU2. Interestingly, downregulation of DLEU2 in CLL has recently been ascribed to DNA methylation of its $\mathrm{CpG}$ islands [22]. Moreover, long intergenic non-coding RNA p21 (lincRNA-p21) and nuclear enriched abundant transcript 1 (NEAT1) were identified as transcriptional targets of TP53 and implicated in the TP53-depedent cell death after DNA damage in CLL [23]. However, apart from DLEU2, lincRNA-21 and NEAT1, the role of lncRNAs in CLL remains unexplored.

Recently, BM742401 has been found to be a tumor suppressor lncRNA inhibiting metastasis, and repression of BM742401 was associated with inferior survivals in patients with gastric cancer [24]. Moreover, restoration of BM742401 resulted in inhibition of metastasis and decreased secretion of extracellular MMP9, which enhances cell migration and invasion. As the promoter region and the entire transcript of BM742401 are embedded in a $\mathrm{CpG}$ island, and the fact that methylation may serve as an alternative mechanism for silencing of tumor suppressor lncRNAs; herein, we postulated $B M 742401$ is a tumor suppressor lncRNA inactivated by DNA methylation in CLL.

\section{RESULTS}

\section{Methylation of BM742401}

\section{Normal controls}

As illustrated in the schematic diagram showing the putative promoter region of BM742401 (Supplementary Figure S1), methylation of BM742401 was studied by MSP and pyrosequencing by primers designed upstream to the transcription start site. The promoter of BM742401 was completely unmethylated in all of the nine normal controls (N1 to N9), but completely methylated in the positive control DNA (Figure 1A). Direct sequencing analysis of the M-MSP products amplified from the positive control showed expected conversion of unmethylated cytosine, but not methylated cytosine, into thymidine, indicating complete bisulfite conversion and specificity of the MSP (Figure 1B).

\section{CLL cell lines}

In CLL cell lines, M-/U-MSP analysis showed that the promoter of BM742401 was completely methylated (MM) in MEC1, I83-E95 and WAC3CD5+, partially methylated (MU) in HG3, and completely unmethylated (UU) in MEC2, 232B4, and CLL-AAT (Figure 1C). Moreover, the MSP methylation statuses $\mathrm{MM}, \mathrm{MU}$, and UU were confirmed by quantitative bisulfite pyrosequencing (Figure 1D). Together, these data suggested that methylation of BM742401 was absent in normal controls but detected in four (57.1\%) CLL cell lines, and hence tumor-specific.

\section{Primary CLL samples at diagnosis}

By MSP, methylation of BM742401 was detected in 43/98 (43.9\%) of diagnostic CLL marrow samples (Figure 1E). Methylation of BM742401 is found significantly associated with older age and higher diagnostic lymphocyte count (Supplementary Table S1). The mean age of patients with and without methylation of BM742401 were 69 and 64 years old respectively $(P=0.03$; Supplementary Table S1). The mean diagnostic lymphocyte count of patients with and without methylation of $B M 742401$ were $29 \times 10^{9} / \mathrm{L}$ and $64 \times 10^{9} / \mathrm{L}$ respectively $(P=0.04$; Supplementary Table $\mathrm{S} 1)$. However, there was no association between the methylation of BM742401 and diagnostic hemoglobin level, platelet count, Rai stage, gender, death or high-risk karyotypes (Supplementary Table S1). The median OS of CLL patients with and without methylation of BM742401 were 97 and 94 months respectively $(P=0.85$; Supplementary Table S1). Moreover, among 44 low-risk CLL patients with $\operatorname{del}(13 \mathrm{q} 14)$, normal karyotype or other karyotypic changes, methylation of BM742401 significantly correlated with advanced Rai stage ( $\geq$ stage 2$)(P=0.002)$, but not survival. In addition, in 51 CLL patients with concomitant methylation study for both BM742401 and miR-129-2, methylation of $B M 742401$ was significantly associated with that of miR-129-2 $(P=0.05)$.

\section{Methylation and expression of $B M 742401$ in CLL cells}

\section{In CLL cell lines}

CLL cell lines methylated for BM742401 were significantly associated with lower expression of $B M 742401$, as evidenced by RT-qPCR showing a higher $\triangle \mathrm{Ct}(\mathrm{Ct} B M 742401-\mathrm{Ct} G A P D H)$, than those CLL cell lines completely unmethylated for $B M 742401(P=0.032)$ (Figure 2A). 


\section{5-AzadC treatment of WAC3CD5+ cells}

In WAC3CD5+ cells, which were completely methylated for $B M 742401$, hypomethylation treatment with 5-AzadC led to the demethylation of BM742401 promoter, as evidenced by the emergence of the U-MSP signal (Figure 2B) and decrease of the mean methylation percentage along the promoter $\mathrm{CpG}$ dinucleotides (Figure 2C) on day 5. Simultaneously, upon 5-AzadC treatment, expression of $B M 742401$ was increased in WAC3CD5+ cells (Figure 2D).

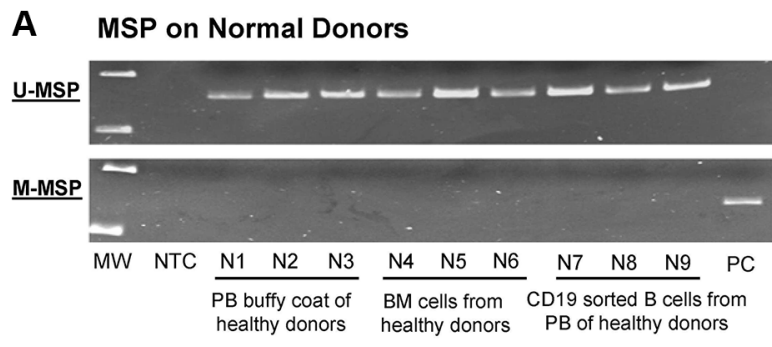

B Direct sequencing of the $B M 742401 \mathrm{M}$-MSP products Wild-type C C C G G G G G G $\underline{C} A \underline{C} C$ G A G $\underline{\mathrm{C} \mathrm{C} \mathrm{C} \mathrm{A} \mathrm{G} \mathrm{G}}$ Methylated $\overline{T T} C$ G G G G G G $\mathrm{T} A \mathrm{~T} C \mathrm{C}$ A G TT T A G

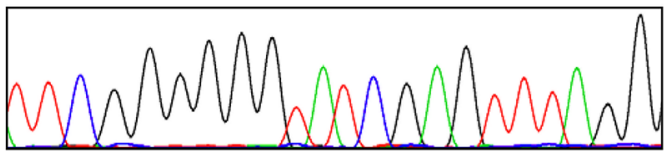

C MSP on CLL cell lines

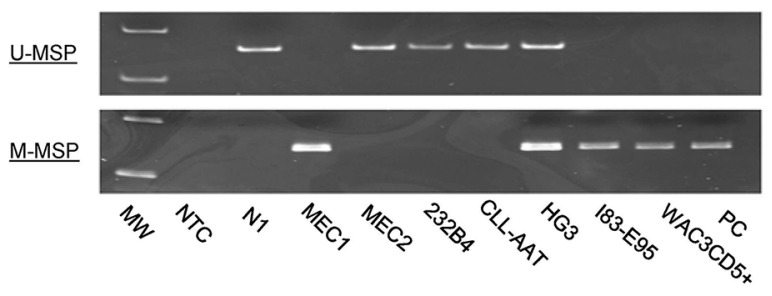

E CLL primary Samples

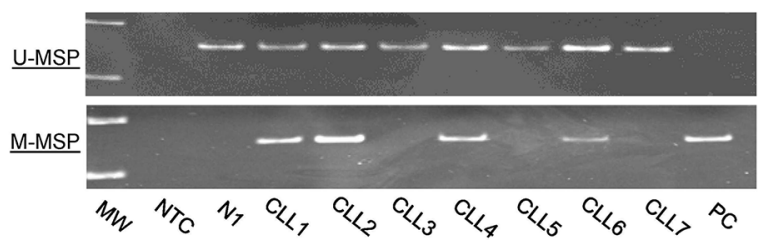

\section{Potential interplay between $B M 742401$ with neighboring GATA6}

While lncRNA may regulate the expression of its neighboring gene [25], whether methylation-mediated silencing of $B M 742401$ is linked to its neighboring protein-coding gene GATA6 was studied in CLL cell lines by qRT-PCR. Results showed that GATA6 had a trend of lower expression in BM742401-methylated cell lines (MEC1, I83-E95 and WAC3CD5, HG3) than BM742401unmethylated cell lines (MEC2, 232B4, and CLL-AAT)

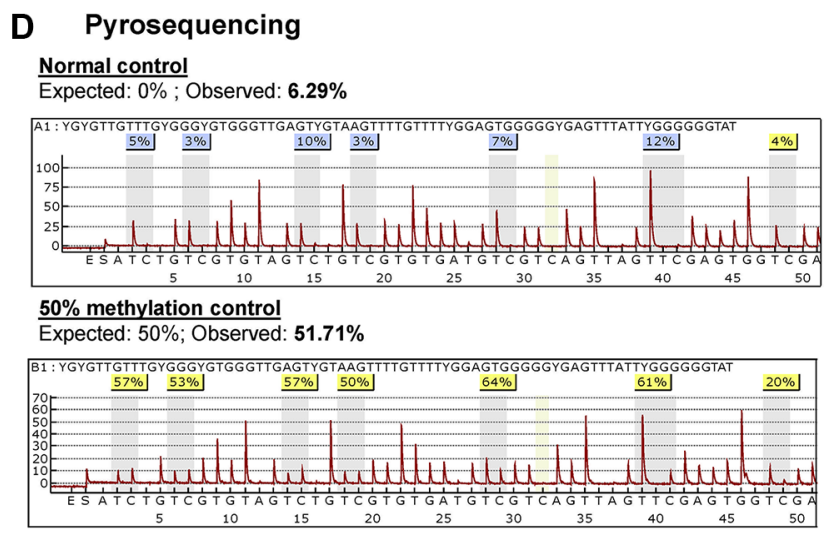

Positive control

Expected: $100 \%$; Observed: $\mathbf{9 6 . 7 1 \%}$
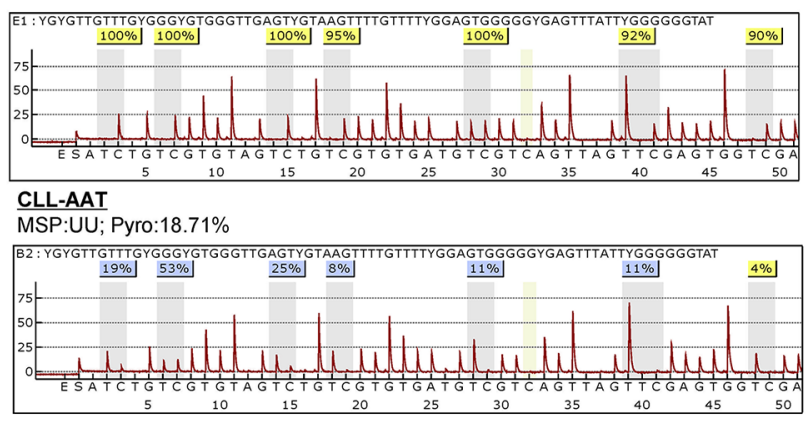

HG3

MSP: UM; Pyro: $71.43 \%$

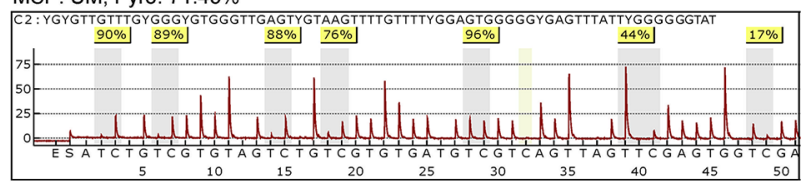

$\underline{183}$

MSP: MM; Pyro: $82.00 \%$

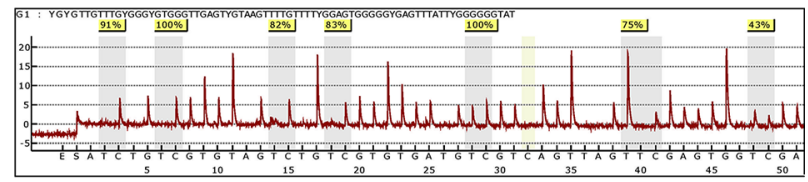

Figure 1: Methylation of BM742401. (A) Methylation-specific PCR (MSP) showed that BM742401 was completely unmethylated in normal controls. (B) Sequencing analysis of the M-MSP product from a methylated positive control showed the expected conversion of unmethylated cytosine to uracil (turned into thymidine after PCR) while leaving methylated cytosine unchanged, indicating the complete bisulfite conversion and specificity of MSP. The unmethylated cytosine was visualized by underlined text while the methylated cytosines highlighted in red. (C) MSP showed the methylation status of BM742401 in a panel of CLL cell lines. (D) Pyrograms of quantitative bisulfite pyrosequencing showed the methylation intensity on a stretch of 7 neighboring $\mathrm{CpG}$ dinucleotides of $B M 742401$ in normal control, $50 \%$ methylation control, positive control with methylated DNA and CLL cell lines with defined MSP methylation status, complete methylation (MM), partial methylation (MU) and unmethylation (UU). (E) MSP showed BM742401 methylation in primary CLL samples. 
$(P=0.14)$ (Supplementary Figure S2), as indicated by a higher $\triangle \mathrm{Ct}(\mathrm{Ct} G A T A 6-\mathrm{Ct} G A P D H)$ in BM742401methylated than $B M 742401$-unmethylated CLL cell lines.

\section{Function of BM742401 in CLL cells}

As BM742401 was frequently methylated in CLL cells, with repressed expression, we postulated that it might act as a tumor suppressive lncRNA. The tumor suppressor function of $B M 742401$ was studied by stable overexpression of BM742401 in WAC3CD5+ CLL cells completely methylated for $B M 742401$ via lentiviral transduction (Figure 3B). By RT-qPCR, BM742401 was confirmed to be overexpressed in WAC3CD5+ cells, as compared with WAC3CD5+ cells stably transduced with empty vector (Figure 3C). Moreover, over-expression of BM742401 resulted in 34\% reduction of cellular proliferation by MTT assay $(P=0.02$, Figure 3D and Supplementary Figure S3A) and 13\% increase of dead cells by Trypan blue exclusion assay $(P=0.0001$, Figure $3 \mathrm{E})$, as well as $11 \%$ increase of apoptotic cells indicated by cells in sub-G1 phase using propidium iodide (PI) staining $(P=0.005$, Supplementary Figure S4A and S4B), indicating that $B M 742401$ is a tumor suppressor in CLL cells. Furthermore, restoration of BM742401 led to increase of the activated, cleaved Caspase 9 and cleaved Caspase 3 (Figure 3F and 3G), but not cleaved Caspase 8 (p43/p41) (data not shown). Moreover, treatment with Z-LEHDFMK, a caspase 9 inhibitor, rescued the percentage of dead cells by $9 \%$ in BM742401-overexpressing cells $(P=0.012$, Supplementary Figure S3), but not in cells with empty vector, confirming the tumor suppressive role of BM742401 is mediated by the intrinsic, Caspase- 9 dependent apoptotic pathway.

\section{A Quantitative RT-PCR}

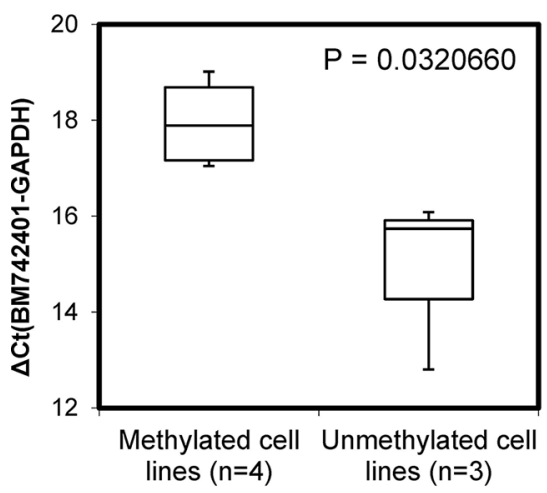

\section{Sequencing analysis of Quantitative RT-PCR products of BM742401}

T T T CA G C G T G G C C G C A T T T G G A A A G G

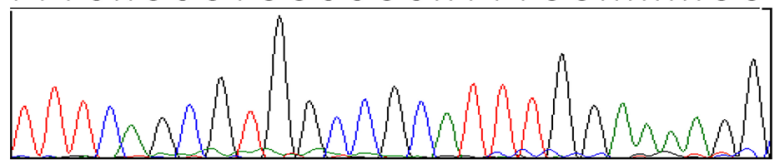

\section{B 5-azadC treatment}

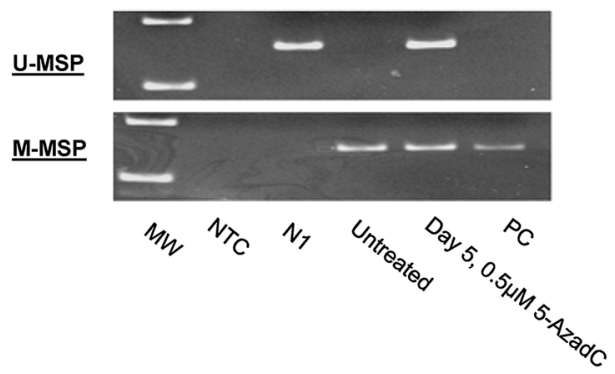

\section{Pyrosequencing}

\section{WAC3CD5+ (5-azadC: Untreated)} MSP: MM; Pyro: $83.43 \%$

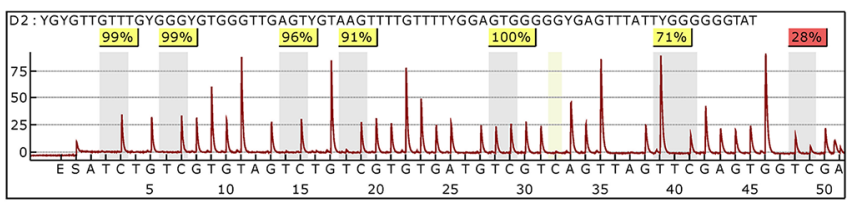

WAC 3 CD5+ (5-azadC: 5 days, $0.5 \mu \mathrm{M})$ MSP: UM; Pyro:48.86\%

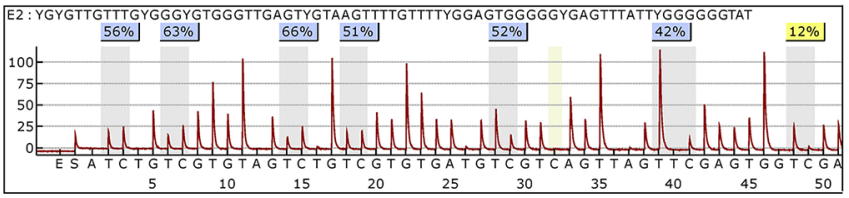

D Quantitative RT-PCR

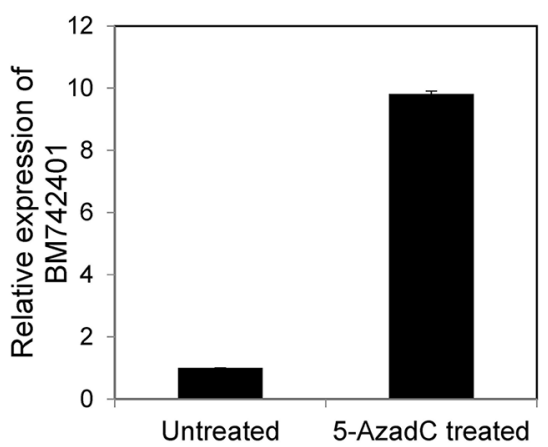

Figure 2: Methylation and expression of BM742401 in CLL cells. (A) Quantitative SYBR Green RT-PCR showed inverse correlation between BM742401 methylation and expression in CLL cell lines. Sequencing analysis of the SYBR Green real-time RT-PCR products for the detection of BM742401. Treatment of CLL cells completely methylated for BM742401 with 5-AzadC, a hypomethylating agent, (B) methylation-specific PCR and (C) quantitative bisulfite pyrosequencing showed BM742401 was demethylated, and (D) quantitative RT-qPCR revealed $B M 742401$ was re-expressed. 


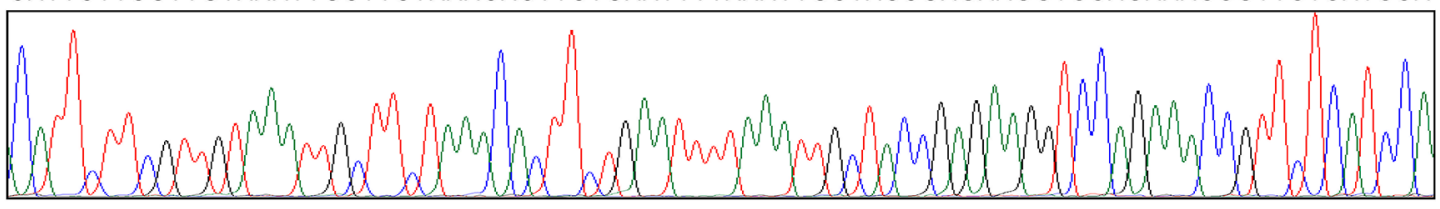

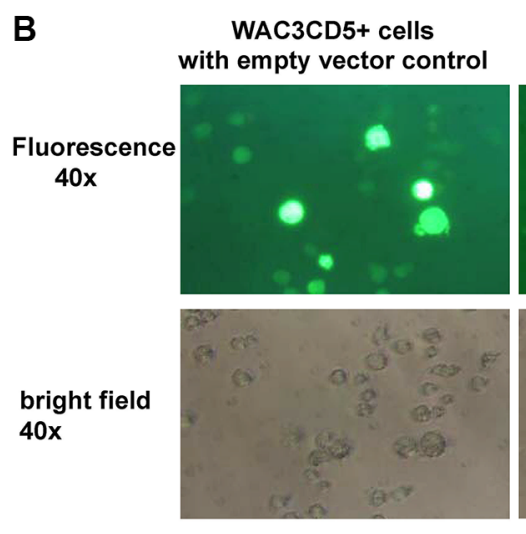

D

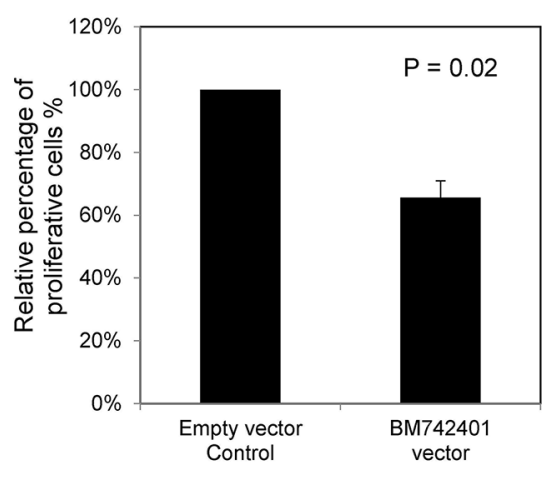

$\mathbf{F}$

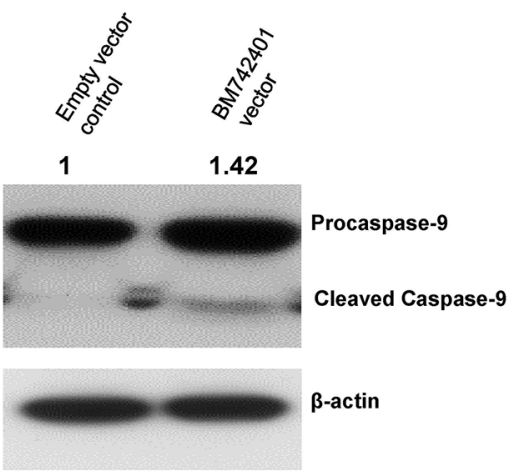

WAC3CD5+ cells with BM742401 vector
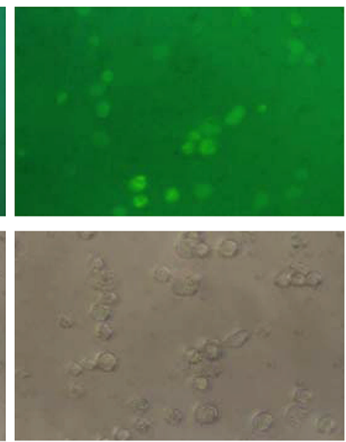

C

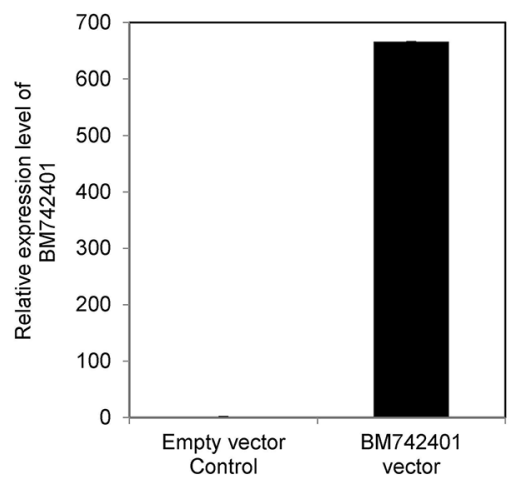

E

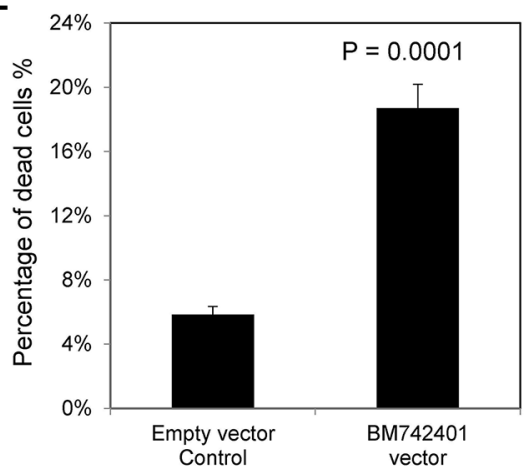

G

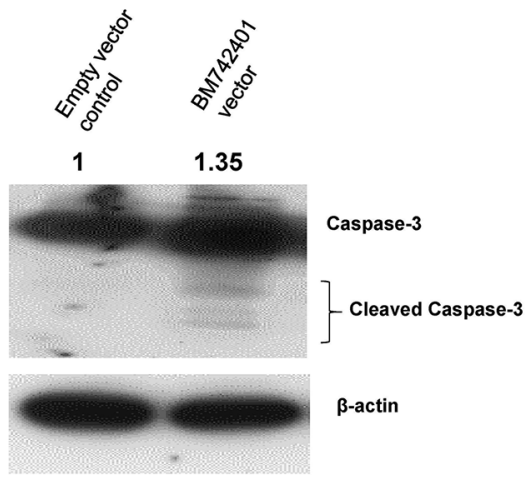

Figure 3: Function of BM742401 in CLL cells. Stable overexpression of BM742401 in WAC3CD5+ CLL cells was performed by lentiviral infection of a GFP-expressing plasmid cloned with full-length BM742401 cDNA. WAC3CD5+ CLL cells stably transduced with GFP-expressing empty plasmid were used as control. (A) Sequence analysis of GFP-expressing plasmid cloned with fulllength BM742401 cDNA. (B) Representative fluorescence (upper, 40× magnification) and bright field images (lower, 40× magnification) of WAC3CD5+ CLL cells stably transfected with empty vector or BM742401 are shown. (C) Quantitative RT-qPCR for BM742401 expression was performed. $\triangle \mathrm{Ct}, \mathrm{Ct} B M 742401$-Ct GAPDH. GAPDH was chosen as reference using the $2^{-\Delta \Delta C \mathrm{~T}}$ method. (D) Relative cell proliferation of CLL cells upon over-expression of BM742401 was measured by MTT assay. (E) Percentage of dead cells was measured by Trypan blue exclusion assay. Data of MTT and Trypan blue assay shown were mean \pm S.D. obtained from experiments in triplicate. Western blot analysis of (F) Caspase 9 and (G) Caspase 3 after BM742401 overexpression. Total protein was extracted the same time after lentiviral transfection and membranes were probed with antibodies to anti-Caspase 3, -Caspase 9 and anti-actin. Signal from anti-actin antibody was used as the endogenous normalizer. The numbers above the bands indicated the relative protein expression measured by densitometric analysis. 
To further confirm the tumor suppressor function of $B M 742401$, the expression of BM742401 in 232B4 cells, which were completely unmethylated for $B M 742401$, was knockdowned by transfection of BM742401specific 2'-O-Methyl phosphorothioate antisense oligonucleotides (2'OMe-PS ASOs). Upon transfection of BM742401-specific ASO1 and ASO2, the expression of $B M 742401$ was reduced by $56 \%$ and $66 \%$ respectively (Supplementary Figure S5A). Moreover, knockdown by BM742401-specific ASO1 led to $19.9 \%$ increase of cell proliferation by MTT assay $(P=0.04)$ (Supplementary Figure S5B) and 6\% decrease of dead cells by Trypan blue exclusion assay ( $P=0.03$ ) (Supplementary Figure S5C), as compared with non-targeting control ASO. Furthermore, BM742401-specific ASO2-mediated depletion of BM742401 led to $8 \%$ decrease of dead cells by Trypan blue exclusion assay $(P=0.03)$ compared with non-targeting control ASO (Supplementary Figure S5C).

\section{DISCUSSION}

Several observations were made in this study. First, this is the first study demonstrating tumor-specific methylation of BM742401 in CLL as shown by frequent methylation in CLL cell lines and primary CLL samples but not normal controls including normal CD19-sorted peripheral B-cells. Moreover, BM742401 expression in CLL cell lines inversely correlated with methylation status. Furthermore, hypomethylating treatment in completely methylated CLL cells led to demethylation and re-expression of $B M 742401$. Hence, methylation of $B M 742401$ promoter is associated with reversible silencing in CLL.

In addition to its frequent methylation in cell lines, BM742401 was frequently methylated in primary CLL samples at diagnosis. Interestingly, there was a significant association of BM742401 methylation with higher lymphocyte counts. Despite the lack of association between BM742401 methylation and survival in CLL patients with low-risk cytogenetic aberrations, BM742401 methylation correlated with the advanced Rai stage ( $\geq$ stage 2 ), which is a poor prognostic factor for CLL. Given the limited number of samples in our cohort, the prognostic impact of BM742401 methylation in CLL warrants further studies. In light of the frequent methylation of BM742401 methylation in primary CLL cells, BM742401 methylation is important in CLL leukemogenesis. This contrasted with the frequent methylation of miR-124-1 in myeloma cell lines but not primary myeloma plasma cells, hence excluding its role in pathogenesis of clinical myeloma [26].

Secondly, to gain insight into its biological function, BM742401 was stably re-expressed in WAC3CD5+ cells with complete methylation of $B M 742401$, which led to inhibition of cellular proliferation and enhanced cell apoptosis, indicating a tumor suppressive role in CLL. Caspases, a family of aspartate-specific cysteine proteases, are crucial mediators of apoptosis [27]. Cellular apoptosis may be activated by extrinsic/death receptor pathway or intrinsic/mitochondrial pathway [27]. In the extrinsic pathway, apoptosis is triggered upon activation of Caspase 8 upon ligation of membrane death receptors, such as members of the tumor necrosis factor (TNF) superfamily or Fas [28]. On the other hand, intrinsic apoptosis is triggered by the activation of Caspase 9 upon mitochondrial release of cytochrome $\mathrm{C}$, resulting in the formation of apoptosome comprising Apaf-1, Caspase 9 and cytochrome $\mathrm{C}$ in the cytoplasm [29]. Once apoptosis is triggered, whether by extrinsic or intrinsic mechanism, both Caspase 8 and Caspase 9 leads to cleavage and hence activation of the effector Caspase 3 to initiate activation of the caspase cascade [30]. Herein, overexpression of BM742401 resulted in the activation of Caspase 9 and Caspase 3, but not Caspase 8, showing that the tumor suppressive function of BM742401 in CLL was mediated by activation of intrinsic apoptosis pathway.

Thirdly, BM742401 gene exists in an antisense orientation to a neighboring protein-coding gene GATA6. Moreover, in CLL cell lines, methylation-mediated silencing of $B M 742401$ was linked to a lower expression of its neighboring protein-coding gene, GATA6. GATA6 is implicated in human cancers as TSGs or oncogenes. In particular, loss-of-function GATA6 in human astrocytes may lead to the acceleration of tumorigenesis, suggesting its tumor suppressor role in astrocytoma [31]. Conversely, GATA6 overexpression could promote pancreatic carcinogenesis as an oncogene by activating the canonical Wnt signaling through antagonizing the expression of DKK1 at mRNA and protein level [32]. Indeed, recent studies showed that lncRNAs could regulate the expression of neighboring genes in cis or trans [25, 33]. For instance, $A N R I L$, an antisense transcript overlapping the INK4b/ARF/INK4a tumor suppressor locus, has been shown to repress its cognate transcript INK4b in cis through recruitment of polycomb repressive complex 1 , and hence INK4b/ARF/INK4a repression [34]. The potential of molecular interaction between $B M 742401$ and its neighboring GATA6 warrants further study.

Last but not least, an interesting observation was that methylation of BM742401, localized to chromosome $18 \mathrm{q} 11.2$ was associated with that of $m i R-129-2$ at chromosome 11q11.2 in CLL patients. Our previous study showed that miR-129-2, which targets sex determining region Y-box 4 (SOX4) and cyclin-dependent kinase 6 $(C D K 6)$, was frequently methylated in CLL at diagnosis $[11,35,36]$. Therefore, concomitant frequent methylation of both BM742401 and miR-129-2 might collaborate to enhance cell proliferation and survival, and hence CLL leukemogenesis. Intriguingly, recent reports have suggested that lncRNAs could modulate their regulatory roles through potential interaction with miRNAs [37]. Therefore, a potential role of $m i R-129-2$ in BM742401-associated tumor suppression in CLL warrants further studies. 
Taken together, this study was first report of $B M 742401$ as a tumor suppressor lncRNA frequently methylated in CLL. Given the tumor suppressive role of BM742401 in CLL, the exact mechanism of tumor suppressive function of $B M 742401$ warrant further studies.

\section{MATERIALS AND METHODS}

\section{Patient samples}

Bone marrow samples were obtained from 98 Chinese CLL patients diagnosed according to the standard morphologic and immunophenotyping criteria as described $[10,38]$. Patient details and demographic data have been reported in our previous study and were listed in Table 1 [13]. The median overall survival (OS) of this cohort was 89 months. The median OS of those with advanced and limited Rai stage were 57 and 111 months respectively $(P=0.006)$. Moreover, the median OS for those with and without high/intermediate-risk karyotype were 43 months and 111 months respectively $(P=0.04)$. Of these, methylation of $m i R-129-2$ has been reported in 51 patients previously [11]. This study was approved by the Institutional Review Board of Queen Mary Hospital and samples were collected with written informed consent and in accordance with the Declaration of Helsinki.

\section{Cell lines and culture}

The human CLL cell lines MEC1 and CLLAAT were purchased from Deutsche Sammlung von Mikroorganismen und Zellkulturen Deutsche $\mathrm{GmbH}$ (DSMZ) (Braunschweig, Germany) and American Type Culture Collection (ATCC) (Manassas, USA) respectively. MEC2 [39] I83-E95 [40] and WAC3CD5+ [40] were kindly provided by Dr John C. Byrd, Department of Medicine, Ohio State University. HG3 and 232B4 were established and kindly provided by Prof. Anders Rosén, Department of Clinical \& Experimental Medicine, Linköping University [41, 42]. Cell cultures were maintained in 90\% RPMI $1640+10 \%$ fetal bovine serum, supplemented with $50 \mathrm{U} / \mathrm{ml}$ penicillin and $50 \mu \mathrm{g} / \mathrm{ml}$ streptomycin (Invitrogen, Carlsbad, CA, USA) in a humidified atmosphere of $5 \% \mathrm{CO}_{2}$ at $37^{\circ} \mathrm{C}$.

\section{Methylation-specific polymerase chain reaction (MSP)}

DNA was extracted from 98 diagnostic marrow samples, seven CLL cell lines and nine healthy normal controls [buffy coats of peripheral blood $(N=3)$, bone marrow $(N=3)$, and CD19-sorted peripheral blood B-cells $(N=3)$ ] by the QIAamp DNA Blood Mini Kit (QIAGEN, Germany). Bisulfite treatment of DNA was performed to convert unmethylated cytosine to uracil (but unaffecting methylated cytosine) using the EpiTect
Bisulfite Kit kit (QIAGEN, Hilden, Germany). Each bisulfite-treated sample was amplified using primer sets specific to methylated DNA (methylated-MSP, M-MSP) and unmethylated DNA (unmethylated-MSP, U-MSP) respectively. Details of primers and conditions for M-MSP and U-MSP of BM742401 were given in Table 2.

\section{Quantitative bisulfite pyrosequencing}

Methylation-unbiased primers were designed by PSQ Assay Design software (Biotage) and used to amplify the promoter region of $B M 742401$, encompassing the amplicon of MSP, in the bisulfite-treated DNA. Forward primer: 5'-AGGGGAGGAGAGAAAAGAG-3'; biotinylated reverse primer: 5'-AACTATACACTACCAAC TCCT-3'; condition: $2 \mathrm{mM} / 61^{\circ} \mathrm{C} / 50 \mathrm{X}$. A stretch of DNA with 7 consecutive $\mathrm{CpG}$ dinucleotides was pyrosequenced using sequencing primer: 5'-GTTTAGGTAGATAA TGAGAGT-3'.

\section{5-Aza-2'-deoxycytidine (5-AzadC) treatment}

WAC3CD5+ cells at log-phase were cultured with $0.5 \mu \mathrm{M}$ of 5-AzadC (Sigma-Aldrich, St. Louis, MO, USA) for 5 days in a six-well plate at a density of $1 \times 10^{6} \mathrm{cell} / \mathrm{s} / \mathrm{ml}$. Fresh 5-AzadC was replaced every 24 hours. Cells treated with 5 -AzadC on day 0 and day 5 were harvested for further analysis.

\section{Plasmid construction and lentiviral transduction of BM742401}

The full-length cDNA of BM742401 was amplified and cloned into the XbaI and EcoRI sites of a pCDHCMV-MCS-EF1-copGFP lentivector (System Biosciences; LV500A-1) (Table 2). The insert sequence was confirmed by direct sequencing (Figure 3A). According to the manufacturer's instructions, the BM742401-containing construct and pPACK packaging plasmid mix were then co-transfected into $293 \mathrm{TN}$ cells, followed by collection of supernatant at 48 hours after transfection. The supernatant $(200 \mu 1)$ was added into WAC3CD5+ cells and cultured for 72 hours. GFP-positive WAC3CD5+ cells were selected by flow cytometry (BD FACSAria I Cell Sorter) and further cultured for two weeks. WAC3CD5+ cells transduced with GFP-expressing empty vector plasmid were used as negative control.

\section{Knockdown of BM742401 by antisense oligonucleotides (ASO)}

BM742401-specific 2'-O-Methyl phosphorothioate antisense oligonucleotides (2' OMe-PS ASOs) were designed, chemically synthesized and purchased from Integrated DNA Technologies (Coralville, IA, USA). ASO1 (BM742401-2'OMe/PS-320): $\mathrm{mG}^{*} \mathrm{~mA}^{*} \mathrm{~mA}^{*} \mathrm{~mA}{ }^{*} \mathrm{~mA}^{*} \mathrm{G}^{*}$ 
Table 1: Demographic data of the 98 CLL patients at diagnosis

\begin{tabular}{lc}
\hline \multicolumn{1}{c}{ Characteristic } & Value \\
\hline Gender & $67(68.4 \%)$ \\
Male & $31(31.6 \%)$ \\
Female & \\
Age & 67 \\
Median & $37-91$ \\
Range & \\
Rai stageł & $56(62.2 \%)$ \\
Limited Stage (stage 0/I/II) & $34(37.8 \%)$ \\
Advanced Stage (stage III/IV) & \\
Lymphocyte count & $16 \times 10^{9} / \mathrm{L}$ \\
Median & $10-540 \times 10 \% / \mathrm{L}$ \\
Range & \\
High-risk cytogenetics* & $3(4.8 \%)$ \\
del(17p) & $2(3.2 \%)$ \\
del(11q) & $11(17.7 \%)$ \\
trisomy 12 & \\
Low-risk cytogenetics* & $19(30.6 \%)$ \\
del(13) & $20(32.4 \%)$ \\
normal karyotype & $7(11.3 \%)$ \\
Other karyotype abnormalities &
\end{tabular}

t. from 90 patients with clinical data; *, from 62 patients with cytogenetic data.

A*A*C*C*A*A*A*A* $\mathrm{A}^{*} \mathrm{mC} \mathrm{C}^{*} \mathrm{~mA} \mathrm{~A}^{*} \mathrm{~mA} \mathrm{~A}^{*} \mathrm{~m} \mathrm{G}^{*} \mathrm{mG}$; ASO2 (BM742401-2'OMe/PS-96): $\mathrm{mG}^{*} \mathrm{mG}^{*} \mathrm{~mA}^{*} \mathrm{mG}^{*} \mathrm{mG}^{*} \mathrm{C}^{*}$ $A^{*} A * A * G^{*} T^{*} G^{*} G^{*} A * A * m C^{*} m G^{*} m U^{*} m A^{*} m A$; nontargeting ASO control: $\mathrm{mG}^{*} \mathrm{mC}^{*} \mathrm{mG}^{*} \mathrm{mU}^{*} \mathrm{~mA} \mathrm{~A}^{*} \mathrm{~T}^{*} \mathrm{~T}^{*} \mathrm{~A} * \mathrm{~T} *$ $\mathrm{A}^{*} \mathrm{G}^{*} \mathrm{C}^{*} \mathrm{C}^{*} \mathrm{G}^{*} \mathrm{~A} * \mathrm{~m} \mathrm{U}^{*} \mathrm{~m} \mathrm{U}^{*} \mathrm{~m} \mathrm{~A}^{*} \mathrm{~m} \mathrm{~A}^{*} \mathrm{mC}$. Knockdown of BM742401 was performed in 232B4 cells, which were plated at a density of $1 \times 10^{6} / \mathrm{ml}$ in a 12 -well plate and transfected with BM742401-ASO1, BM742401-ASO2 or non-targeting control ASO at a final concentration of 10nM using Lipofectamine 2000 (Invitrogen), according to the manufacturer's instructions. Cells were cultured and analyzed at 48 hours after transfection. The efficiency of knockdown was determined by qRT-PCR of BM742401.

\section{Quantification of BM742401}

Total RNA was extracted by mirVana miRNA Isolation Kit (Ambion, Austin, TX, USA) and reverse transcribed by QuantiTect Reverse Transcription Kit (QIAGEN, Valencia, CA). BM742401 was quantified using SYBR Green Master Mix (ABI, Foster City, CA, USA), following the manufacturer's instructions. The $2^{-\Delta \Delta \mathrm{CT}}$ method was used to detect the expression of
BM742401 before and after BM742401 over-expression (or 5-AzadC treatment) in WAC3CD5+ cells [43]. The $\triangle \mathrm{CT}$ method is used for comparing the BM742401 expression between methylated and unmethylated CLL cell lines. GAPDH was used as endogenous control for data analysis of $B M 742401$. Primers and conditions used for detection of BM742401 were summarized in Table 2.

\section{MTT assay, Trypan blue dye exclusion assay and cell cycle analyses}

The tumor suppressor function of BM742401 was studied by MTT and Trypan blue dye exclusion assay. In brief, cells from each stably transduced sample were seeded in a 96-well microtitre plate at $2 \times 10^{5}$ cells/well in $100 \mu \mathrm{l}$ of medium. After 72 hours, each well was added $10 \mu \mathrm{l}$ of $5 \mathrm{mg} / \mathrm{ml}$ MTT reagent (Sigma-Aldrich) and incubated for four hours. Then each well was added with $100 \mu \mathrm{l}$ dimethyl sulfoxide (DMSO), followed by the measurement of absorbance at $550 \mathrm{~nm}$ with reference to $650 \mathrm{~nm}$. The percentage of dead cells was measured by Trypan blue dye exclusion assay under microscope. Five random microscopic fields were counted for each 
Table 2: Primer sequences and reaction condition

Gene

Forward primer $\left(5^{\prime}\right.$ to $\left.3^{\prime}\right)$

Reverse primer $\left(5^{\prime}\right.$ to $\left.3^{\prime}\right)$

$\mathrm{Tm} / \mathrm{cycles} / \mathrm{MgCl}_{2}$

References

(I) Methylation-specific PCR (MSP)

BM742401

U-MSP

TGTGTTGTTTAGGTAGATAAT

GAGAGTTGT

CCAAATCAAACATTCT

ATAACCTCCA

$60^{\circ} \mathrm{C} / 35 \times / 2 \mathrm{mM}$

M-MSP

CGTTTAGGTAGATAATGAGA

GTCGC

AAATCAAACGTTCTAT

$62^{\circ} \mathrm{C} / 35 \times / 1.5 \mathrm{mM}$

(II) Reverse transcription-polymerase chain reaction (RT-PCR)

BM742401

GAPDH
TTGCTACCGAGAAGGTCCAG

ACCACAGTCCATGCCATCACT
GACATCCTTGTAGAAA

AGAACCAA

TCCACCACCCTGTTG

CTGTA $60^{\circ} \mathrm{C} / 40 \times$

$60^{\circ} \mathrm{C} / 40 \times$

(III) Cloning PCR

BM742401

GCTCTAGACATTCTTCGTTG
TAAATTGCTTCTAA

GCTCTAGACGGAATTC AAAAGGAGCAATCAC TTATTTTTCC $56^{\circ} \mathrm{C} / 40 \times / 2 \mathrm{mM}$

Abbreviations: M-MSP, MSP for the methylated allele; U-MSP, MSP for the unmethylated allele; Tm, annealing temperature.

sample. Dead cells $(\%)=($ total number of dead cells per microscopic field / total number of cells per microscopic field) $\times 100$. Cell cycle analysis was conducted by propidium iodide (PI) staining. Briefly, cells were washed in phosphate buffered saline (PBS), fixed in cold $70 \%$ ethanol at $4{ }^{\circ} \mathrm{C}$ overnight, washed twice in PBS, resuspended and then incubated in $50 \mu \mathrm{g} / \mathrm{ml}$ PI staining solution with $5 \mu \mathrm{g} / \mathrm{ml} \mathrm{RNase} \mathrm{A} \mathrm{at} 4^{\circ} \mathrm{C}$ for at least 2 hours, followed by the analyze of flow cytometry (Beckman Coulter Cytomics FC 500). Each sample was done in duplicate. MTT assay and Trypan blue dye exclusion assay were performed in triplicates of each sample. Data were plotted by mean \pm standard deviation and compared by Student's $t$-test.

\section{Treatment with Caspase 9 inhibitor}

WAC3CD5+ cells transduced with GFP-expressing empty vector or BM742401 vector were treated with $10 \mu \mathrm{M}$ Z-LEHD-FMK (Caspase 9 inhibitor, R\&D system) for 3 days in a 12 -well plate at a density of $1 \times 10^{6}$ cells $/ \mathrm{ml}$ respectively. After 72 hours, empty or BM742401 vector cells with or without treatment were harvested for Trypan Blue exclusive assay.

\section{Western blotting}

Seventy-two hours after equal number of cells $\left(2 \times 10^{6}\right)$ from stably transduced samples were seeded in a six-well plate, total proteins $(20 \mu \mathrm{g})$ were isolated at for Western Blot analysis, as described [10]. The primary antibodies incubated at $4^{\circ} \mathrm{C}$ overnight were anti-Caspase 3 (1:1000; Cell Signaling), -Caspase 8 (1:1000; Cell Signaling), -Caspase 9 (1:1000; Cell Signaling) and anti-actin (1:5000; Sigma-Aldrich, USA), followed by incubation with anti-rabbit or anti-mouse horseradish peroxidase conjugate secondary antibody at room temperature for 1 hour. ECL plus Western blotting detection reagent was used for the detection of protein signals with X-ray film (Amersham Biosciences, Buckinghamshire, UK). Protein bands were quantified using densitometry as measured by Quantity One 4.6.2 software (Bio-Rad); hence relative protein expression was expressed in comparison to corresponding control.

\section{Statistical analysis}

In 98 primary CLL samples, the correlation between BM742401 methylation with continuous (mean age, mean lymphocyte counts, diagnostic hemoglobin or platelet counts) and categorical variables (gender, Rai stage or high-risk karyotypes) were analyzed by student's $t$-test and chi-square test (or Fisher's exact test) respectively. $\mathrm{OS}$ is measured from the date of diagnosis to the date of last follow-up or death. Survival was plotted by the Kaplan-Meier method and compared by the log-rank test. OS of CLL patients with limited Rai stage (stage 0/I/II) was compared with those with advanced Rai stage (stage III/IV). Moreover, OS of CLL patients with high-risk karyotypes [del(17p), del(11q) or trisomy 12] was compared to those with standard-risk karyotypes [del(13q), normal karyotype or other karyotypic changes]. 
Association between methylation of $B M 742401$ and $m i R$ $129-2$ was studied by chi-square test. All $P$ values were two-sided.

\section{ACKNOWLEDGMENTS}

We would like to thank Dr John Byrd, Department of Medicine, Ohio State University, USA for the CLL cell lines (MEC2, WAC3CD5+ and I83-E95) and Prof. Anders Rosén, Department of Clinical \& Experimental Medicine, Linköping University for the CLL cell lines (HG3 and 232B4). Moreover, we thank Dr Seon-Young Kim, Medical Genomics Research Center, KRIBB, Daejeon, Republic of Korea \& Department of Functional Genomics, University of Science and Technology, Daejeon, Republic of Korea for the generous provision of plasmid of pcDNA3.1(+)-BM742401.

\section{CONFLICTS OF INTEREST}

The authors declare no conflicts of interest.

\section{FUNDING}

The University of Hong Kong Seed Funding Programme for Basic Research (Ref: 201511159202).

\section{REFERENCES}

1. Esteller M. Epigenetics in cancer. N Engl J Med 2008; 358:1148-1159.

2. Oakes CC, Claus R, Gu L, Assenov Y, Hüllein J, Zucknick M, Bieg M, Brocks D, Bogatyrova O, Schmidt CR, Rassenti L, Kipps TJ, Mertens D, et al. Evolution of DNA Methylation Is Linked to Genetic Aberrations in Chronic Lymphocytic Leukemia. Cancer Discov. 2014; 4:348-361.

3. Herman JG, Civin CI, Issa J-PJ, Collector MI, Sharkis SJ, Baylin SB. Distinct patterns of inactivation of p15INK4B and p16INK4A characterize the major types of hematological malignancies. Cancer Res. 1997; 57:837-841.

4. Gonzalez-Zulueta M, Bender CM, Yang AS, Nguyen TD, Beart RW, Van Tornout JM, Jones PA. Methylation of the $5^{\prime} \mathrm{CpG}$ island of the p16/CDKN2 tumor suppressor gene in normal and transformed human tissues correlates with gene silencing. Cancer Res. 1995; 55:4531-4535.

5. Chim CS, Pang R, Fung TK, Choi CL, Liang R. Epigenetic dysregulation of Wnt signaling pathway in multiple myeloma. Leukemia. 2007; 21:2527-2536.

6. Chim CS, Fung TK, Wong KF, Lau JS, Liang R. Frequent DAP kinase but not p14 or Apaf-1 hypermethylation in B-cell chronic lymphocytic leukemia. J Hum Genet. 2006; 51:832-838.

7. Wang LQ, Kwong YL, Wong KF, Kho CS, Jin DY, Tse E, Rosèn A, Chim CS. Epigenetic inactivation of mir-34b/c in addition to mir-34a and DAPK1 in chronic lymphocytic leukemia. J Transl Med. 2014; 12:52.

8. Chen SS, Claus R, Lucas DM, Yu L, Qian J, Ruppert AS, West DA, Williams KE, Johnson AJ, Sablitzky F. Silencing of the inhibitor of DNA binding protein 4 (ID4) contributes to the pathogenesis of mouse and human CLL. Blood. 2011; 117:862-871.

9. Chim CS, Pang R, Liang R. Epigenetic dysregulation of the Wnt signalling pathway in chronic lymphocytic leukaemia. J Clin Pathol. 2008; 61:1214-1219.

10. Wang LQ, Kwong YL, Kho CSB, Wong KF, Wong KY, Ferracin M, Calin GA, Chim CS. Epigenetic inactivation of miR-9 family microRNAs in chronic lymphocytic leukemia-implications on constitutive activation of $\mathrm{NF \kappa B}$ pathway. Mol Cancer. 2013; 12:173.

11. Wong KY, Yim RLH, Kwong YL, Leung CY, Hui PK, Cheung F, Liang R, Jin DY, Chim CS. Epigenetic inactivation of the MIR129-2 in hematological malignancies. J Hematol Oncol. 2013; 6:16.

12. Chim CS, Wong KY, Leung CY, Chung LP, Hui PK, Chan SY, Yu L. Epigenetic inactivation of the hsa-miR-203 in haematological malignancies. J Cell Mol Med. 2011; 15:2760-2767.

13. Wang LQ, Wong KY, Rosèn A, Chim CS. Epigenetic silencing of tumor suppressor miR-3151 contributes to Chinese chronic lymphocytic leukemia by constitutive activation of MADD/ERK and PIK3R2/AKT signaling pathways. Oncotarget. 2015; 6:44422-44436. doi: 10.18632/oncotarget.6251.

14. Mercer TR, Dinger ME, Mattick JS. Long non-coding RNAs: insights into functions. Nat Rev Genet. 2009; 10:155-159.

15. Prensner JR, Chinnaiyan AM. The emergence of lncRNAs in cancer biology. Cancer Discov. 2011; 1:391-407.

16. Esteller M. Non-coding RNAs in human disease. Nat Rev Genet. 2011; 12:861-874.

17. Ponting CP, Oliver PL, Reik W. Evolution and functions of long noncoding RNAs. Cell. 2009; 136:629-641.

18. Gupta RA, Shah N, Wang KC, Kim J, Horlings HM, Wong DJ, Tsai MC, Hung T, Argani P, Rinn JL. Long non-coding RNA HOTAIR reprograms chromatin state to promote cancer metastasis. Nature. 2010; 464:1071-1076.

19. Zhou Y, Zhang X, Klibanski A. MEG3 noncoding RNA: a tumor suppressor. J Mol Endocrinol. 2012; 48:R45-R53.

20. Zhang X, Zhou Y, Mehta KR, Danila DC, Scolavino S, Johnson SR, Klibanski A. A pituitary-derived MEG3 isoform functions as a growth suppressor in tumor cells. J Clin Endocrinol Metab. 2003; 88:5119-5126.

21. Calin GA, Dumitru CD, Shimizu M, Bichi R, Zupo S, Noch E, Aldler H, Rattan S, Keating M, Rai K, Rassenti L, Kipps T, Negrini M, et al. Frequent deletions and downregulation of micro-RNA genes miR15 and miR16 at 13q14 in chronic lymphocytic leukemia. Proc Natl Acad Sci USA. 2002; 99:15524-15529. 
22. Garding A, Bhattacharya N, Claus R, Ruppel M, Tschuch C, Filarsky K, Idler I, Zucknick M, Caudron-Herger M, Oakes C. Epigenetic upregulation of lncRNAs at 13q14. 3 in leukemia is linked to the In Cis downregulation of a gene cluster that targets NF-kB. PLoS Genet. 2013; 9:e1003373.

23. Blume C, Hotz-Wagenblatt A, Hüllein J, Sellner L, Jethwa A, Stolz T, Slabicki M, Lee K, Sharathchandra A, Benner A. p53-dependent non-coding RNA networks in chronic lymphocytic leukemia. Leukemia. 2015; 29:2015-2023.

24. Park SM, Park SJ, Kim HJ, Kwon OH, Kang TW, Sohn HA, Kim SK, Noh SM, Song KS, Jang SJ. A known expressed sequence tag, BM742401, is a potent lincRNA inhibiting cancer metastasis. Exp Mol Med. 2013; 45:e31.

25. Petruk S, Sedkov Y, Riley KM, Hodgson J, Schweisguth F, Hirose S, Jaynes JB, Brock HW, Mazo A. Transcription of bxd noncoding RNAs promoted by trithorax represses Ubx in cis by transcriptional interference. Cell. 2006; 127:1209-1221.

26. Wong KY, So CC, Loong F, Chung LP, Lam WW, Liang R, Li GK, Jin DY, Chim CS. Epigenetic Inactivation of the miR-124-1 in Haematological Malignancies. PloS one. 2011; 6:e19027.

27. Cullen S, Martin S. Caspase activation pathways: some recent progress. Cell Death Differ. 2009; 16:935-938.

28. Lin Y, Devin A, Rodriguez Y, Liu Z-g. Cleavage of the death domain kinase RIP by caspase- 8 prompts TNFinduced apoptosis. Genes Dev. 1999; 13:2514-2526.

29. Zou H, Li Y, Liu X, Wang X. An APAF-1 cytochrome c multimeric complex is a functional apoptosome that activates procaspase-9. J Biol Chem. 1999; 274:11549-11556.

30. Porter AG, Jänicke RU. Emerging roles of caspase-3 in apoptosis. Cell Death Differ. 1999; 6:99-104.

31. Kamnasaran D, Qian B, Hawkins C, Stanford WL, Guha A. GATA6 is an astrocytoma tumor suppressor gene identified by gene trapping of mouse glioma model. Proc Natl Acad Sci USA. 2007; 104:8053-8058.

32. Zhong Y, Wang Z, Fu B, Pan F, Yachida S, Dhara M, Albesiano E, Li L, Naito Y, Vilardell F. GATA6 activates Wnt signaling in pancreatic cancer by negatively regulating the Wnt antagonist Dickkopf-1. PLoS One. 2011; 6:e22129.

33. Andersson R, Gebhard C, Miguel-Escalada I, Hoof I, Bornholdt J, Boyd M, Chen Y, Zhao X, Schmidl C, Suzuki T. An atlas of active enhancers across human cell types and tissues. Nature. 2014; 507:455-461.

34. Yap KL, Li S, Muñoz-Cabello AM, Raguz S, Zeng L, Mujtaba S, Gil J, Walsh MJ, Zhou MM. Molecular interplay of the noncoding RNA ANRIL and methylated histone H3 lysine 27 by polycomb CBX7 in transcriptional silencing of INK4a. Mol Cell. 2010; 38:662-674.
35. Anderlind C, Spira A, Cardoso WV, Lü J. miR-129 regulates cell proliferation by downregulating Cdk6 expression. Cell Cycle. 2010; 9:1809-1818.

36. Huang Y, Liu J, Deatherage D, Luo J, Mutch D, Goodfellow P, Miller D, Huang T. Epigenetic repression of microRNA-129-2 leads to overexpression of SOX4 oncogene in endometrial cancer. Cancer Res. 2009; 69:9038-9046.

37. Jalali S, Bhartiya D, Lalwani MK, Sivasubbu S, Scaria V. Systematic transcriptome wide analysis of lncRNA-miRNA interactions. PloS one. 2013; 8:e53823.

38. Hallek M, Cheson BD, Catovsky D, Caligaris-Cappio F, Dighiero G, Döhner H, Hillmen P, Keating MJ, Montserrat E, Rai KR, Kipps TJ. Guidelines for the diagnosis and treatment of chronic lymphocytic leukemia: a report from the International Workshop on Chronic Lymphocytic Leukemia updating the National Cancer Institute-Working Group 1996 guidelines. Blood. 2008; 111:5446-5456.

39. Stacchini A, Aragno M, Vallario A, Alfarano A, Circosta P, Gottardi D, Faldella A, Rege-Cambrin G, Thunberg U, Nilsson K. MEC1 and MEC2: two new cell lines derived from B-chronic lymphocytic leukaemia in prolymphocytoid transformation. Leuk Res. 1999; 23:127-136.

40. Wendel-Hansen V, Sällström J, De Campos-Lima P, Kjellström G, Sandlund A, Siegbahn A, Carlsson M, Nilsson K, Rosen A. Epstein-Barr virus (EBV) can immortalize B-cll cells activated by cytokines. Leukemia. 1994; 8:476-484.

41. Rosén A, Bergh AC, Gogok P, Evaldsson C, Myhrinder AL, Hellqvist E, Rasul A, Björkholm M, Jansson M, Mansouri L. Lymphoblastoid cell line with B1 cell characteristics established from a chronic lymphocytic leukemia clone by in vitro EBV infection. OncoImmunology. 2012; 1:18-27.

42. Lanemo Myhrinder A, Hellqvist E, Bergh A-C, Jansson M, Nilsson K, Hultman P, Jonasson J, Buhl AM, Bredo Pedersen L, Jurlander J. Molecular characterization of neoplastic and normal "sister" lymphoblastoid B-cell lines from chronic lymphocytic leukemia. Leukemia \& Lymphoma. 2013; 54:1769-1779.

43. Livak KJ, Schmittgen TD. Analysis of Relative Gene Expression Data Using Real-Time Quantitative PCR and the $2{ }^{\Delta \Delta}$ CT Method. Methods. 2001; 25:402-408.

44. Chim CS, Wong KY, Qi Y, Loong F, Lam WL, Wong LG, Jin DY, Costello JF, Liang R. Epigenetic inactivation of the miR-34a in hematological malignancies. Carcinogenesis. 2010; $31: 745-750$. 